\title{
Pulmonary dissemination of tumor cells after extended resection of thyroid carcinoma with cardiopulmonary bypass
}

\author{
Seiki Hasegawa, MD, Yosuke Otake, MD, Toru Bando, MD, Hiroyuki Cho, MD, Kenji Inui, MD, and Hiromi Wada, MD,
} Kyoto, Japan

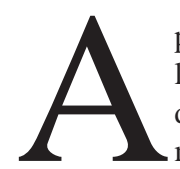

pplication of cardiopulmonary bypass (CPB) to oncologic surgery is still controversial. One of the possible disadvantages of CPB application to operations for malignancies is hematogenous dissemination of tumor cells. ${ }^{1}$ However, to our knowledge, no such case has been reported. ${ }^{2}$ Here we describe a case of intrapulmonary tumor spread after surgical resection of intrathoracic extension of thyroid papillary carcinoma with $\mathrm{CPB}$.

\section{Clinical Summary}

A 78-year-old Japanese woman was admitted to Kyoto University Hospital, with severe and uncontrollable pain in the right side of the neck. A mass extending from the right side of the neck into the right internal jugular vein, superior vena cava, and right atrium was revealed by means of computed tomographic scanning, magnetic resonance imaging, ultrasonography, and fluorodeoxyglucose-positron emission scanning, but no definite diagnosis was obtained. No intrapulmonary lesions were noted on preoperative chest radiography and computed tomographic scanning. Because of persistent pain and moderate heart failure, the patient underwent semiemergency surgery. Intrathoracic extension of papillary thyroid carcinoma was diagnosed by means of intraoperative frozen section. En bloc resection of the thyroid tumor, the right subclavian vein, the superior vena cava, and part of the right atrium was performed by using CPB through a median sternotomy. CPB was performed with cannulation of the ascending aorta, left innominate vein, and inferior vena cava. The duration of CPB was 27 minutes, with transfusion of $640 \mathrm{~mL}$ of concentrated red blood corpuscles.

From the Department of Thoracic Surgery, Kyoto University, Kyoto, Japan.

Received for publication March 14, 2002; accepted for publication March $24,2002$.

Address for reprints: Seiki Hasegawa, MD, Associate Professor, Department of Thoracic Surgery, Kyoto University, 53 Shogoin, Kyoto 606-8507,

Japan (E-mail: seikiha@kuhp.kyoto-u.ac.jp).

J Thorac Cardiovasc Surg 2002;124:635-6

Copyright (C) 2002 by The American Association for Thoracic Surgery

$0022-5223 / 2002 \$ 35.00+0 \quad \mathbf{1 2 / 5 4 / 1 2 5 0 6 0}$

doi: $10.1067 / \mathrm{mtc} .2002 .125060$

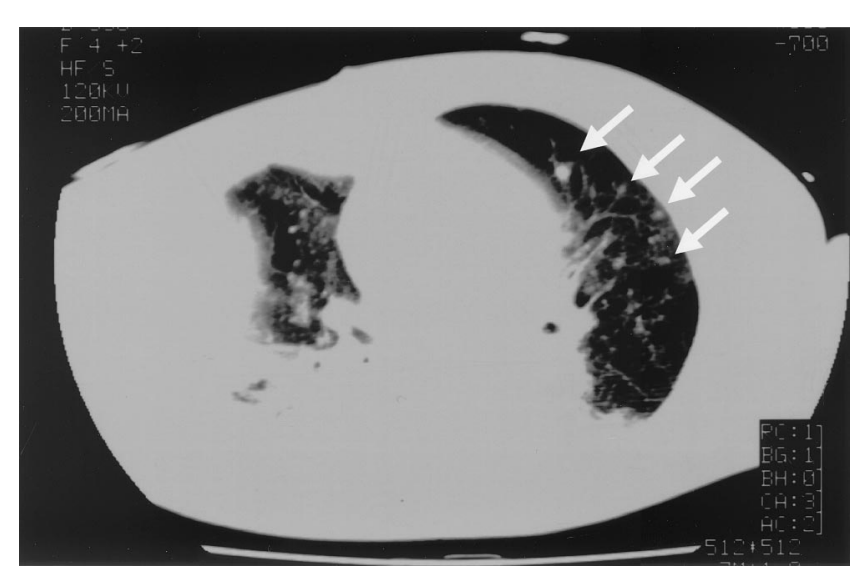

Figure 1. Chest computed tomographic scan on POD 13 revealed bilateral multiple pulmonary nodules (arrows) with bilateral infiltration. Thyroid carcinoma cells were detected in bronchoalveolar lavage fluid on POD 24.

The surgical margins were examined with intraoperative frozen sections and confirmed to be free of tumor cells.

The patient was extubated on postoperative day (POD) 1 but required reintubation on POD 3 as a result of airway mucus retention. Two weeks after the operation, bilateral infiltration with nodular shadows became evident (Figure 1). Pseudomonas aeruginosa was detected in a bronchoscopy specimen obtained on POD 14. Thyroid carcinoma cells were detected in bronchoalveolar lavage fluid from the left $\mathrm{B}^{3}$ bronchus on POD 24. The patient died on POD 36 as a result of respiratory failure. Autopsy was not performed.

\section{Comments}

There are 2 possible mechanisms through which CPB might contribute to hematogenous dissemination of tumor cells.

First, tumor cells contaminated in the reservoir blood might spread through the arterial cannula. Akchurin et $\mathrm{al}^{3}$ found that in patients who underwent combined oncologic and cardiovascular surgery, tumor cells were seen only on the internal (machine) 
surface but not the outer (patient) surface of the filters, and they concluded that CPB might be used without increasing the risk of hematogenous tumor dissemination. However, the above mechanism could still conceivably operate in particular situations, such as a long CPB period, re-use of suctioned blood, and intravascular extension of the tumor. Because blood transfusion is believed to be a risk factor in oncologic surgery, surgeons tend to prefer re-use of suctioned blood rather than transfusion. In the present case intravascular tumor growth was seen in the superior vena cava. Furthermore, suctioned blood from the surgical field was re-used through the Cell-Saver System (Compact Advanced Wash Set BT745C/125; Dideco s.p.a., Mirandola, Italy).

Second, modification of homeostasis by CPB may liberate tumor cells that have already been spread preoperatively but the growth or migration of which has been suppressed by the host defense system. Boldt and associates ${ }^{4}$ reported that circulating adhesion molecules were increased during cardiac surgery but remained unchanged in long-duration abdominal or lung operations.
Although not proven conclusively, it is strongly suspected that $\mathrm{CPB}$ played a significant role in the early postoperative tumor dissemination seen in the present case, which illustrates a possible disadvantage of CPB use in oncologic surgery. Our current policy for application of CPB to cancer surgery is strict patient selection and no re-use of suctioned blood.

\section{References}

1. Peters RM, Swain JA. Management of the patient with emphysema, coronary artery disease, and lung cancer. Am J Surg. 1982;143:701-5.

2. Gillinov AM, Greene PS, Stuart RS, Heitmiller RF. Cardiopulmonary bypass as an adjunct to pulmonary surgery. Chest. 1996;110:571-4.

3. Akchurin RS, Davidov MI, Partigulov SA, et al. Cardiopulmonary bypass and cell-saver technique in combined oncologic and cardiovascular surgery. Artif Organs. 1997;21:763-5.

4. Boldt J, Kumle B, Papsdorf M, Hempelmann G. Are circulating adhesion molecules specifically changed in cardiac surgical patients? Ann Thorac Surg. 1998;65:608-14. 